Si-95-2

\title{
The short-time Dynamics of the Critical Potts Model
}

\author{
L. Schülke and B. Zheng \\ Universität-GH Siegen, D-57068 Siegen, Germany
}

March 1995

\begin{abstract}
The universal behaviour of the short-time dynamics of the three state Potts model in two dimensions at criticality is investigated with Monte Carlo methods. The initial increase of the order is observed. The new dynamic exponent $\theta$ as well as exponent $z$ and $\beta / \nu$ are determined. The measurements are carried out in the very beginning of the time evolution. The spatial correlation length is found to be very short compared with the lattice size.
\end{abstract}

PACS: 64.60.Ht, 02.70.Lq, 05.70.Jk, 82.20.Mj 
for years it has been known that there exist universality and scaling behaviour for statistical systems at criticality in equilibrium or near equilibrium, more or less due to the infinite spatial and time correlation length. Recently it has been observed that universality and scaling may also be present far from equilibrium. One of the examples is that the Ising model, initially in very high temperature, is suddenly quenched to the critical temperature and then evolves with the dynamics of model A. According to an argument of Janssen et al. with two-loop $\epsilon$-expansion [1], besides the well-known long-time universal behaviour, there exists another universal stage in the earlier time, termed critical initial slip, which sets in right after the microscopic time scale. The characteristic behaviour of such process is that, if a non-zero but small initial magnetization $m_{0}$ is generated in the system, the anomalous dimension of the operator $m_{0}$ gives rise to the critical increase of the magnetization

$$
M(t) \sim m_{0} t^{\theta}
$$

with $\theta$ being a new dynamic critical exponent. Detailed scaling analysis reveals [2] that the characteristic time scale for the critical initial slip is $t_{0} \sim m_{0}^{-z / x_{0}}$, where $x_{0}$ is the scaling dimension of $m_{0}$, and related to $\theta$ by $x_{0}=\theta z+\beta \nu$. Interesting enough, it was pointed out that the exponents $\beta, \nu$ and $z$ should be valued the same as those in the equilibrium or long-time stage of the relaxation. Previously $\theta$ has been measured with Monte Carlo simulation in two dimensions somehow indirectly from the power law decay of the autocorrelation [3, 4], and recently in three diemensions directly from the power law increase of the magnetization [5]. They are in good agreement with the result from the $\epsilon$-expansion. Furthermore, based on the scaling relation in the initial stage of the time evolution, a new promising way for measuring the exponents $z, \beta$ and $\nu$ from the finite size scaling has been proposed [6]. This indicates a possible broad application of the shorttime dynamics. Therefore more and deeper understanding of this phenomenon becomes urgent.

As far as we know, even though analytical perturbative calculations for the critical initial slip can be extended to the $O(N)$ vector model [1, 2], the numerical results are so far limited to the Ising model [3, 4, 7, 5, 6]. The purpose of this letter is to report systematic Monte Carlo simulations of the short-time behaviour of the two dimensional Potts model at criticality relaxing from high temperature states. A refined measurement of the exponent $\theta, z$ and $\beta / \nu$ from the power law behaviour of the physical observables is presented. It relates the above mentioned indirect and direct measurements of $\theta$ each other and provides a consistent test of the scaling relation for the Potts model. Finite size effects and the week dependence of the measurement of $\theta$ on $m_{0}$ are discussed and the spatial correlation length is computed.

From the scaling analysis, the autocorrelation has the initial behaviour

$$
A(t) \sim t^{-d / z+\theta}
$$


Figure 1: Magnetization $M(t) / M(0)$ vs. time in double-log scale for the initial value $m_{0}=0.08$.

in case of $m_{0}=0$. Most of the previous measurements of $\theta$ were based on this relation. A disadvantage is that one has to input $z$ to obtain $\theta$. Since $z$ is one order of magnitude bigger than $\theta$, a small relative error of $z$ will induce a big error in $\theta$. This becomes more severe when $\theta$ is getting smaller. A direct measurement from the power law increase of the order parameter can improve this situation [5]. As we will see later, for the Potts model we did observe the same initial increase of the order shown in (1). The week dependence of the practical measurement of $\theta$ on $m_{0}$ appears, however, more visible in the Potts model than in the Ising model. Therefore in this letter we consider a correction of $\theta$ for finite $m_{0}$. Since $\theta$ for the Potts model is smaller than that for the 2D Ising model, its measurement from the auto-correlation is harder.

On the other hand, traditionally the dynamic exponent $z$ is defined and measured from the long-time exponential decay of the time correlation or the magnetization of the systems [8, 9]. Due to the critical slowing down this is somehow difficult. From the above discussion, however, it is easy to realize that with $\theta$ in hand, one can obtain $z$ quite rigorously from the power law decay (2) of the autocorrelation. Since the measurement is carried out at the beginning of the time evolution, it is efficient. This is an alternative way to measure $z$ from the short-time behaviour of the system. Compared with the method proposed in [6], the advantage is that the measurement can be carried out in a single lattice rather than by comparing two lattices. Finally the static exponent $\beta / \nu$ can be obtained from the power law increase of the second moment

$$
M^{(2)}(t) \sim t^{(d-2 \beta / \nu) / z} .
$$

Since $2 \beta / \nu$ is one order of magnitude smaller than $z$, its measurement is quite sensitive to the error of $z$.

Figure 2: Autocorrelation $A(t)$ vs. time in double-log scale for $L=9,18,36$, 72,144 and 288.

For the study of the short-time dynamics, usually quite a big lattice is used. In this letter, the finite size effects will be discussed. It turns out that for the measurement of $\theta$ the finite size effect is not so big. Furthermore, the spatial correlation is measured and found to be very small compared with the lattice size. This indicates that the mechanism for the universality and scaling in short-time dynamics should be different from that in the equilibrium or near equilibrium.

The Hamiltonian for the $q$ state Potts model

$$
H=J \sum_{<i j>} \delta_{\sigma_{i}, \sigma_{j}}, \quad \sigma_{i}=1, \ldots, q
$$


Figure 3: Second moment $M^{(2)}(t) / M^{(2)}(1)$ vs. time in double-log scale.

with $\langle i j\rangle$ representing nearest neighbors. In this letter we only consider the three state case. It is well known that for the three state Potts model the critical point locates at $J_{c}=\log (1+\sqrt{ } 3)$. As in case of the Ising model, initially the Potts model is prepared to be in a random state with a sharp magnetization $m_{0}$. Then it is released to the evolution with the heat-bath algorithm at the critical temperature. We measure the time evolution of the magnetization, the second moment, the auto-correlation and the spatial correlation respectively

$$
\begin{gathered}
M(t)=\frac{3}{2} \frac{1}{N}\left\langle\sum_{i}\left(\delta_{\sigma_{i}(t), 1}-\frac{1}{3}\right)\right\rangle, \\
M^{(2)}(t)=\frac{9}{4} \frac{1}{N^{2}}\left\langle\left[\sum_{i}\left(\delta_{\sigma_{i}(t), 1}-\frac{1}{3}\right)\right]^{2}\right\rangle, \\
A(t)=\frac{1}{N}\left\langle\sum_{i}\left(\delta_{\sigma_{i}(0), \sigma_{i}(t)}-\frac{1}{3}\right)\right\rangle, \\
C(x, t)=\frac{1}{N}\left\langle\sum_{i}\left(\delta_{\sigma_{i}(t), \sigma_{i+x}(t)}-\frac{1}{3}\right)\right\rangle,
\end{gathered}
$$

where the average is taken over the independent intial configurations. Except for the magentization $M(t)$, the above definitions are restricted here to the case of $m_{0}=0$. In spite of the lack of the analytic analysis, we assume that all the scaling properties including the increase of the order for the Ising model are valid also for the Potts model and test them by numerical simulation. In the same time the related critical exponents will be determined.

In Fig. 1, as an example, the time evolution of the magnetization with the initial value $m_{0}=0.08$ for different lattice size $L$ is displayed in a double logscale to present the power law increase. It is remarkable that the power law increase starts also from the very beginning of the time evolution $t=1$ as it is in the three dimensional Ising model. $\theta$ can be estimated from the slope of the curves. It is clearly seen that $\theta$ converges to a definite value when the lattice size $L \geq 36$. In other words, for the measurement of $\theta$ the finite size effect is already quite small for a lattice size $L=36$. In comparison to this, to observe the power law decay for the auto-correlation one needs much bigger lattices, as will be seen later. In Tab. $1, \theta$ for $L=72$ measured from $t=1$ to $t=15$ for different initial magnetizations has been summarized. The total number of samples for the independent initial configurations is 80,000 for bigger $m_{0}$ and 480, 000 for smaller $m_{0}$. The errors are estimated by dividing the data into four or six groups, respectively. Different from the Ising model, the measured $\theta$ shows slightly more dependence on $m_{0}$. Therefore, according to its definition, a linear 


\begin{tabular}{|c|c|c|c|c|c|}
\hline$m_{0}$ & 0.10 & 0.08 & 0.06 & 0.04 & 0.00 \\
\hline$\theta$ & $0.1076(08)$ & $0.1036(12)$ & $0.0980(06)$ & $0.0925(14)$ & $0.0815(27)$ \\
\hline
\end{tabular}

Table 1: The measured $\theta$ for $L=72$.

extrapolation of $\theta$ to the fixed point $m_{0}=0$ is carried out. This leads to the value

$$
\theta=0.0815(27)
$$

\begin{tabular}{|c|r|r|r|r|}
\hline$L$ & 72 & 144 & 288 & $\infty$ \\
\hline$-d / z+\theta$ & $-0.8510(08)$ & $-0.8387(09)$ & $-0.8335(09)$ & $-0.8283(20)$ \\
$(d-2 \beta / \nu) / z$ & $0.7921(11)$ & $0.7881(14)$ & $0.7875(28)$ & $0.7878(16)$ \\
\hline
\end{tabular}

Table 2: Results for $L=72,144$ and 288 .

Now we set $m_{0}=0$ and measure the auto-correlation. In Fig. 2, the dependence of the auto-correlation on $L$ is presented. Obviously at $L=36$ no power law behaviour is observed. The convergence to a power law behaviour only starts around $L=144$. It is clear that the regime presenting power law grows when the lattice size increases. It is interesting that, somehow different from $M(t)$, the first time steps apparently deviate slightly from the power law. In Tab. 2 the corresponding values for $-d / z+\theta$ measured from $t=5$ to $t=50$ are given. We stop the measurement at $t=50$ due to the obvious bigger finite size effect and statistical errors. The total samples for $L=144$ is 40,000 and for $L=288$ is 16,000. if we only intend to obtain $z$ by taking $\theta$ as input, we should already be satisfied with the lattice size since the results from $L=144$ and $L=288$ are already so close. However, in order to get the better $\beta / \nu$ later by inputting the $z$ measued here, we perform the linear extrapolation for $-d / z+\theta$ over $1 / L$ to $L=\infty$ and obtain

$$
z=2.1983(81)
$$

Compared to the values of $z$ distributed between $z=2.2$ and $z=2.7$ from different numerical measurments [11, 12, 13, 14], our result supports the relative small $z$ [11, 12,

In Fig. 3, the power law increase of the second moment $M^{(2)}(t)$ is shown. The measurement of $(d-2 \beta / \nu) / z$ from $t=5$ to $t=50$ are also listed in Tab. 2. From the data we can see that for $L=144$ and $L=288$, the finite size effects are already less prominent than the statistical errors. Therefore the value $0.7878(22)$ of $(d-2 \beta / \nu) / z$ at $L=\infty$ is only a simple average of them. Here we get

$$
2 \beta / \nu=0.2682(73)
$$


which is in good agreement with the exact value $4 / 15 \approx 0.2667$ [10]. Such coincidence provides a strong support for scaling in the short-time dynamics.

Finally we have also measured the correlation length $\xi(t)$ from the spatial correlation function $C(x, t)$. for example, for $L=288, \xi(t=96) \approx 6.0$ and it is much smaller than the lattice size $L$.

In conclusion, by observing the power law behaviour of the magnetization $M(t)$, the second moment $M^{(2)}(t)$ and the auto-correlation $A(t)$, we confidently confirm the scaling properties for the Potts model in the short-time dynamics and obtain the related critical exponents $\theta, z$ and $\beta / \nu$. This is the first measurment of

$\theta$ for the Potts model. Our way to determine $z$ is efficient. Such an investigation for models in other universality classes should be carried out.

Acknowledgement: The authors would like to thank K. Untch for the help in maintiaining our Workstations.

\section{References}

[1] H. K. Janssen, B. Schaub, and B. Schmittmann, Z. Phys. B 73, 539 (1989).

[2] H. W. Diehl and U. Ritschel, J. Stat. Phys. 73, 1 (1993)

[3] D. A. Huse,Phys. Rev. B40, 304 (1989).

[4] K. Humayun and A. J. Bray, J. Phys. A: Math. Gen. 24, 1915 (1991).

[5] Z. B. Li, U. Ritschel, and B. Zheng, J. Phys. A: Math. Gen. 27, L837 (1994).

[6] Z. B. Li, L. Schülke, and B. Zheng, Phys. Rev. Lett. 74, 3396 (1995)

[7] N. Menyhárd, J. Phys. A: Math. Gen. 27, 663 (1994).

[8] J. K. William, J. Phys. A: Math. Gen. 18, 49 (1985).

[9] S. Wansleben and D. P. Landau, Phys. Rev. B 43, 6006 (1991).

[10] R. J. Baxter, Exactly Solved Models in Statistical Mechanics, (Academic Press, 1982).

[11] M. Aydin and M. C. Yalabik, J. Phys. A: Math. Gen. 18, 1741 (1985).

[12] S. Tang and D. P. Landau, Phys. Rev. B 36, 567 (1987).

[13] K. Binder, J. Stat. Phys. 24, 69 (1981).

[14] M. Aydin and M. C. Yalabik, J. Phys. A: Math. Gen. 21, 769 (1988). 


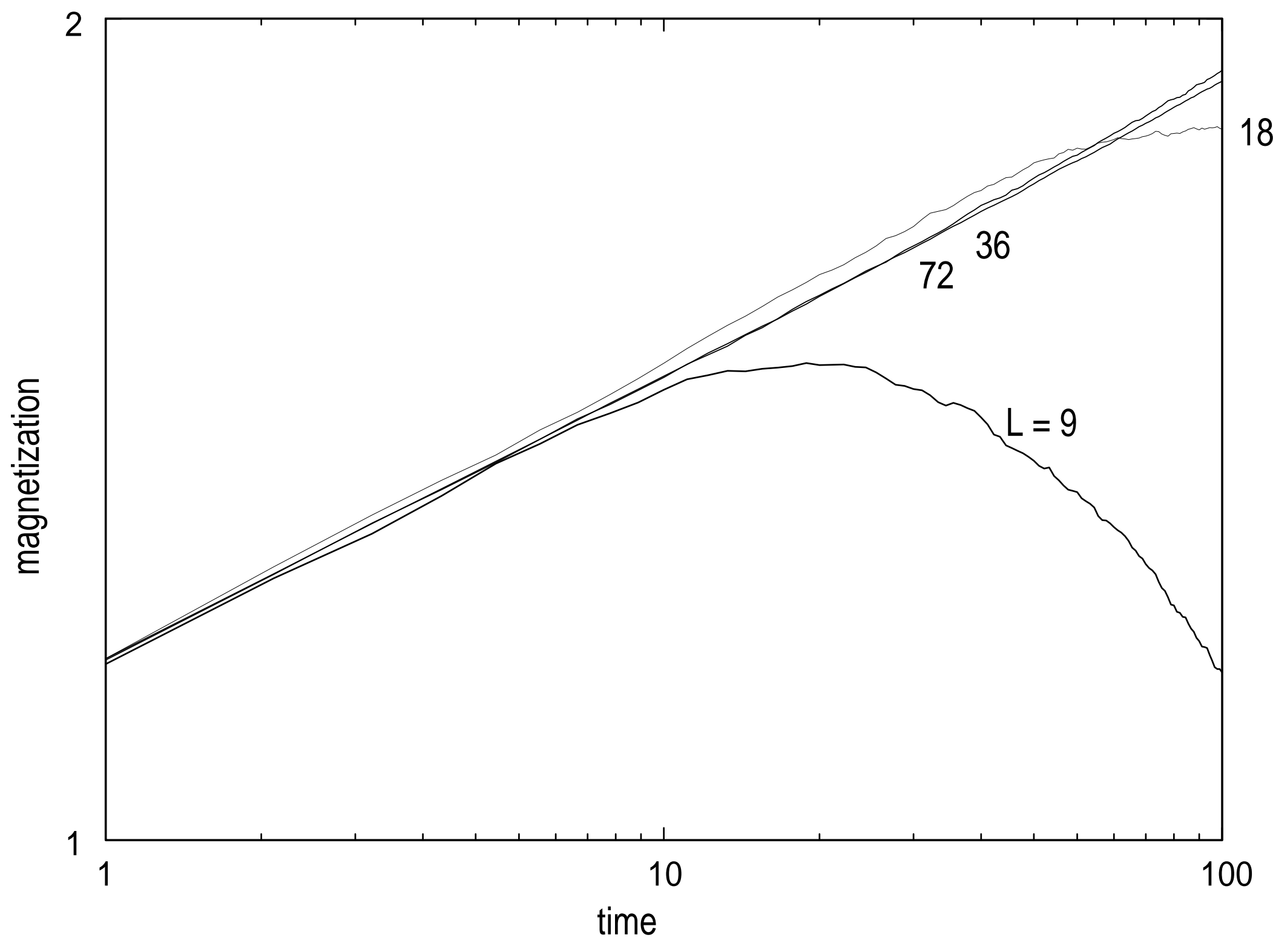




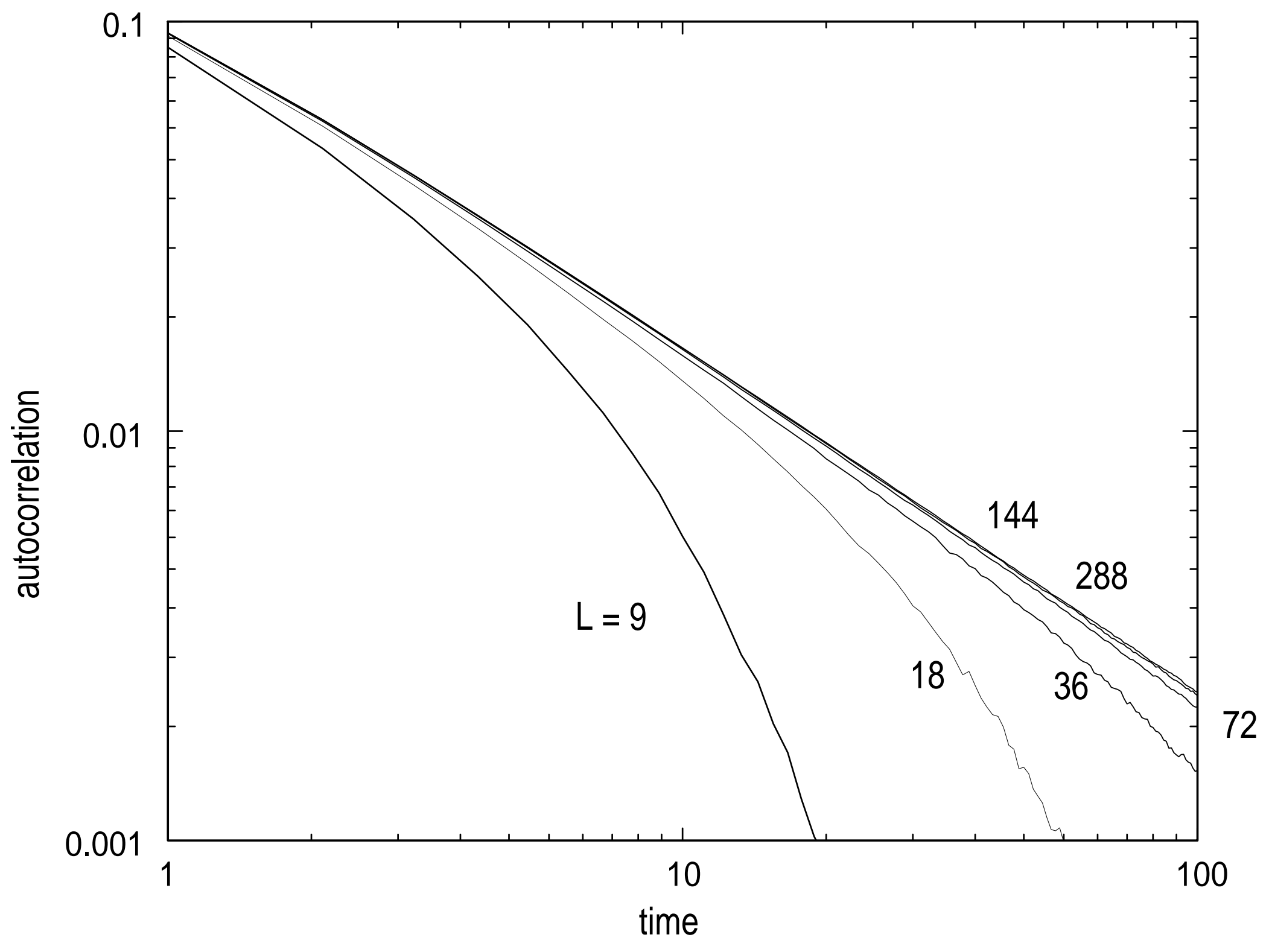




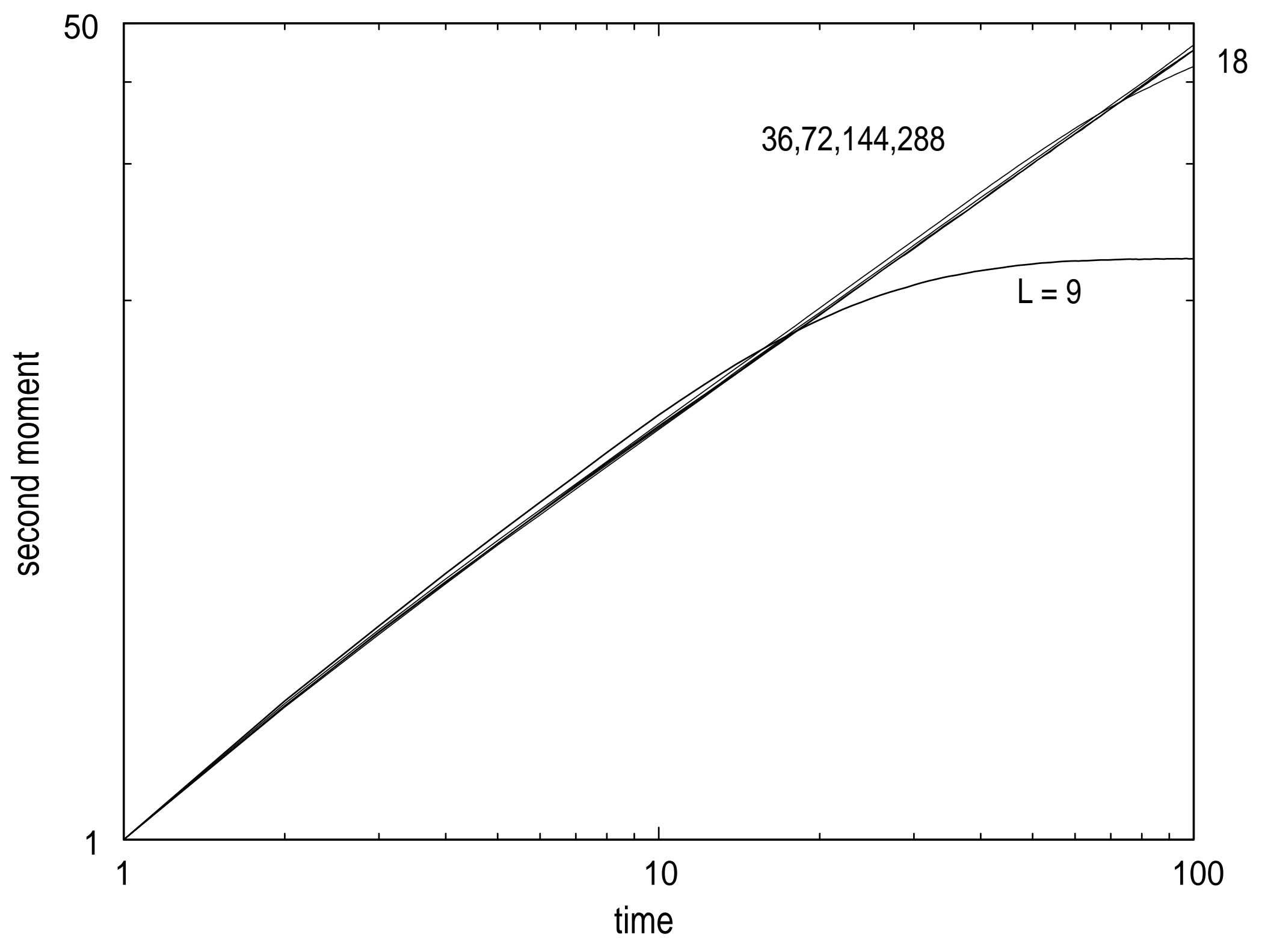

